\title{
A solution for revision rhinoplasty with thinned nasal tip skin: An extended dermofat graft
}

\author{
Daiwon Jun, Na Rim Kim, \\ Young Chul Suh, Jung Ho Lee \\ Department of Plastic and Reconstructive \\ Surgery, Bucheon St. Mary's Hospital, \\ College of Medicine, The Catholic \\ University of Korea, Bucheon, Korea
}

The authors send special thanks to Sammy Jun for an artistic illustration of an extended dermofat graft.
The emphasis on tip plasty in Asian rhinoplasty has led to heightened concerns about complications regarding nasal tip skin. Although uncommon, these complications can cause tremendous frustration to surgeons when encountered. Conventional methods such as skin graft or healing by secondary intention cause an unpleasant scar. Instead, an extended dermofat graft in revision rhinoplasty enables both replacement of the dorsal implant and adequate coverage of the nasal tip without modifying the previous tip plasty. A patient who experienced nasal tip skin thinning after augmentation rhinoplasty was treated successfully with an extended dermofat graft. The authors believe that this is a solid method for managing patients with nasal tip skin complications.

Keywords Rhinoplasty / Esthetics / Nose deformities

\section{INTRODUCTION}

With the ever-increasing demand for a higher nasal tip in Asian rhinoplasty, numerous surgical techniques have been introduced [1]. Consequently, complications involving the nasal tip are encountered more often [2]. Especially in revision rhinoplasty where the skin envelope covering the nasal tip has been compromised, surgeons are hesitant to retain the previous projection. Therefore, it would be ideal to identify a technique for retaining the previously achieved nasal projection and providing adequate coverage. The authors propose a method for achieving this goal utilizing a dermofat graft.

\section{CASE REPORT}

A 23-year-old male patient underwent augmentation rhinoplasty with a septal extension graft and a Gore-Tex implant insertion. Af-

Received: Sep 9, 2020 Revised: Oct 14, 2020 Accepted: Nov 16, 2020 Correspondence: Jung Ho Lee Department of Plastic and Reconstructive Surgery, Bucheon St. Mary's Hospital, College of Medicine, The Catholic University of Korea, 327 Sosa-ro, Wonmi-gu, Bucheon 14647, Korea Tel: +82-32-340-7059, Fax: +82-32-340-2544, E-mail: tfm0822@catholic.ac.kr Copyright @ 2021 The Korean Society for Aesthetic Plastic Surgery. This is an Open Access article distributed under the terms of the Creative Commons Attribution Non-Commercial License (https://creativecommons.org/licenses/by-nc/4.0/) which permits unrestricted non-commercial use, distribution, and reproduction in any medium, provided the original work is properly cited. $\quad w w w . e-a a p s . o r g$ ter 1 month, the cartilage contour of the nasal tip could be observed through thinned skin due to excessive pressure (Fig. 1). Revision rhinoplasty was planned 2 months after the primary rhinoplasty. The length from the nasal root to tip was measured $(5 \mathrm{~cm})$. A dermofat graft was designed in the sacral midline with a longitudinal length that corresponded to the previous measurement. The graft was designed in an elliptical shape $(5 \times 2 \mathrm{~cm})$ with an elongation on one end that would later be used for tip coverage (Fig. 2). The extended dermofat graft was designed to provide both replacement of the dorsal implant and sufficient coverage for the nasal tip (Fig. 3). During secondary rhinoplasty, implant removal was performed, and the graft was inserted without modification of the previous tip plasty. The graft was fixed at two points: one point was sutured at the infratip area and the other was fixated with a bolster suture on the nasal root that was removed 5 days postoperatively. Favorable wound healing was achieved (Fig. 4).

\section{DISCUSSION}

Rhinoplasty is one of the most frequently performed aesthetic surgical procedures in Asians, in whom the nose has several distinctive features, such as a short columella, flared nostril shape, wide alar base, poorly defined nose tip, and low dorsum [3]. The primary goal of Asian rhinoplasty is to achieve a definite contour by increasing the dorsal height and tip projection [4]. At the same time, the surgical outcomes should be naturally harmonized with other parts of the face. 

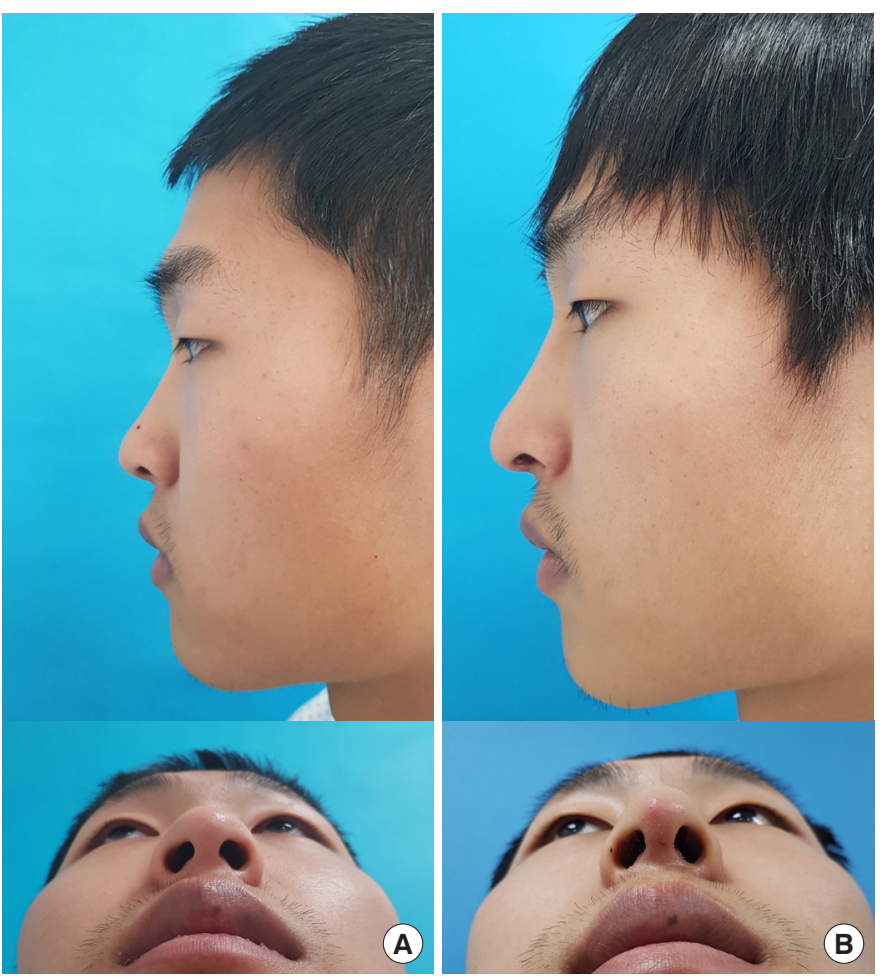

Fig. 1. Outcomes after primary rhinoplasty. Photographs of the patient's preoperative status (A) and 1 month after primary rhinoplasty (B) are shown. Although both the appropriate dorsal augmentation and tip height were achieved, the cartilage contour was visible through the thinned nasal tip skin.
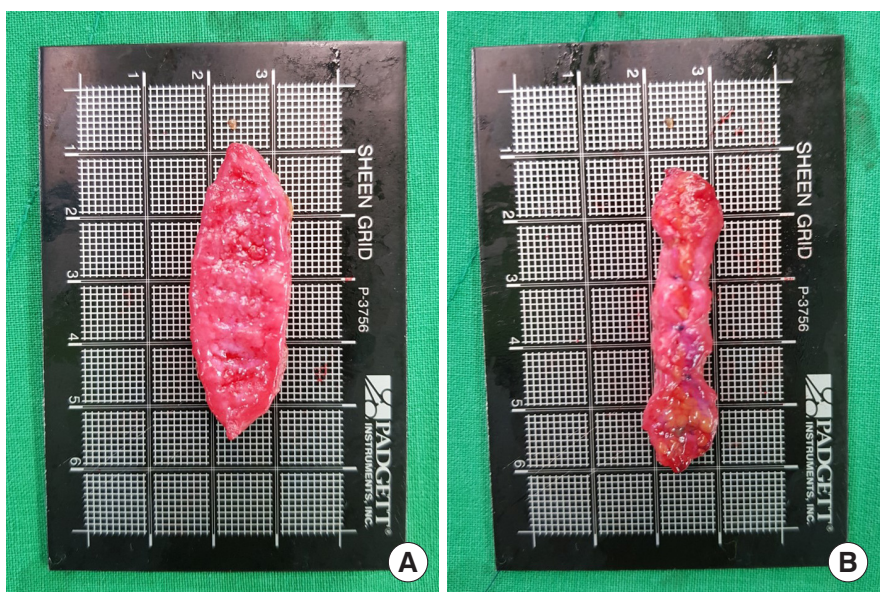

Fig. 2. Intraoperative photographs of extended dermofat graft were taken. The graft was harvested $(\mathrm{A})$ and fabricated with an elongated tail (B) to provide sufficient coverage on nasal tip.

The thickness of the nasal skin affects both the outcomes and the complication risk after surgery [5]. Anatomically, the nasal skin is thickest in the radix and supratip area and thinnest at the rhinion [6]. However due to excessive tip plasty, skin-related complications

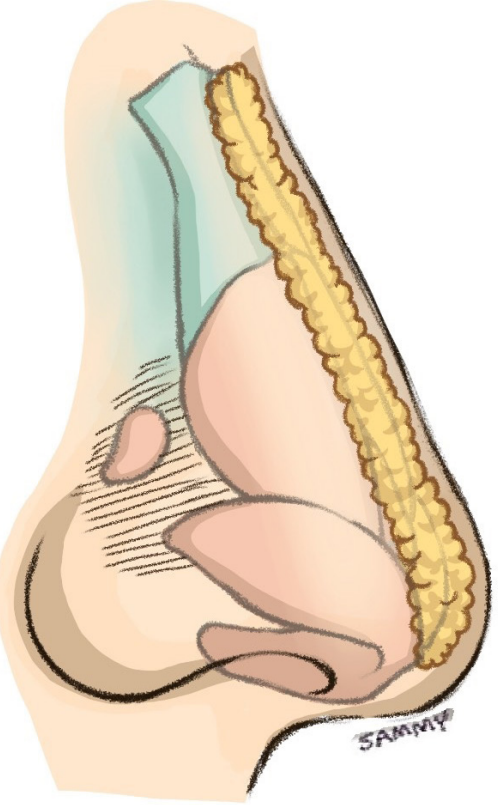

Fig. 3. An illustration of an inserted extended dermofat graft. The graft spans from the nasal root to nasal tip. The measurements were made in advance.
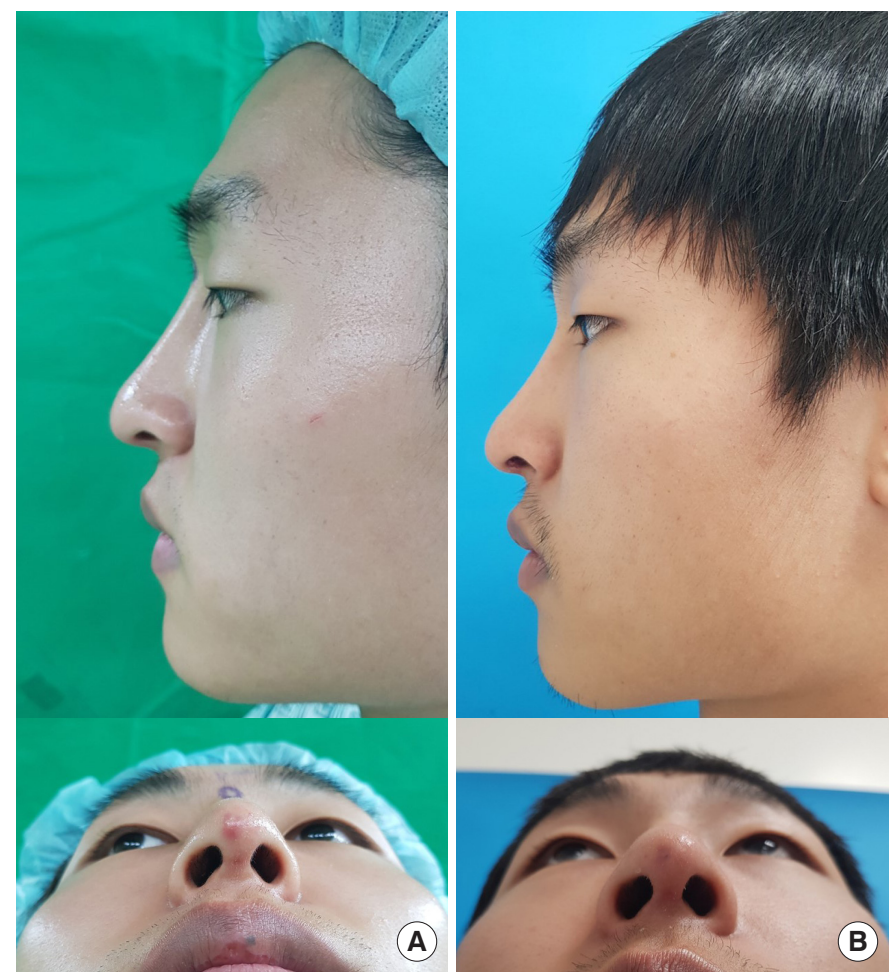

Fig. 4. Outcomes after revision rhinoplasty. Photographs taken 2 months after primary rhinoplasty (A) and 1 month after revision rhinoplasty (B) are shown. Compared to a synthetic implant, a defined dorsal line was sacrificed. However, favorable wound healing in the nasal tip with the maintenance of nasal projection was achieved. 
after rhinoplasty can be commonly observed in the nasal tip [7]. These complications could cause implant infection and/or extrusion, leading to an irreversible misconfiguration. Although uncommon, managing tip necrosis after rhinoplasty is troublesome [8]. Without adequate skin coverage, tip plasty in rhinoplasty is futile. When revision rhinoplasty is required due to a loss of tip skin integrity, surgeons are forced to reduce the projection.

The increasing popularity of augmentation rhinoplasty has made revision rhinoplasty necessary in increasingly many cases. It is widely acknowledged that secondary rhinoplasty is far more complicated than primary rhinoplasty since only limited surgical options remain. From the perspective of the surgeon, it is a complicated problem to solve, since the desired outcome must be achieved while minimizing the risk of failure. For this reason, selection of an ideal material is a prerequisite for revision rhinoplasty.

Dermofat, an autologous dorsal augmentation material, has distinct advantages. First, sufficient quantities can be obtained from anywhere in the body. In addition, the graft can be easily fixated, and its rapid interaction with the surrounding soft tissue makes displacement less likely [9]. Although it is inferior to synthetic implants in terms of its intrinsic strength and long-term volume maintenance, a dermofat graft is still an effective option in situations where synthetic material cannot be used. Numerous studies have reported favorable outcomes of dermofat grafts, which can be an excellent option, especially for secondary rhinoplasty $[10,11]$.

Dermofat grafts are mainly obtained from the sacral area, where thick dermal tissue can be harvested. Traditionally, dermofat grafts are designed in an elliptical shape with a length equal to that from the nasion to the nasal tip. The cranial and caudal ends are fixated over the nasion and nasal tip with the pull-out suture method. In an open approach, the caudal end can be sutured to the soft tissue of the nasal tip [12]. We have designated our method an "extended" dermofat graft because it has an elongated tail that covers the entire nasal tip up to the infratip area. Thus, it can be performed without modifying the previous tip plasty. As a result, the extended dermofat graft can provide both a replacement for a dorsal implant and soft tissue coverage in the nasal tip, while retaining its previous height. The extended dermofat graft is an optimal substitute for secondary rhinoplasty, especially in cases of damage to the tip skin and soft tissue envelope. The relatively short-term follow-up is a limitation of this study.

\section{NOTES}

\section{Conflict of interest}

No potential conflict of interest relevant to this article was reported.

\section{Ethical approval}

The study was exempted from ethical approval (exemption approval No. HC20ZASI0111).

\section{Patient consent}

The patient provided written informed consent for the publication and the use of his images.

\section{ORCID}

Daiwon Jun

Na Rim Kim

Young Chul Suh

Jung Ho Lee

\section{REFERENCES}

1. Lee KC, Kwon YS, Park JM, et al. Nasal tip plasty using various techniques in rhinoplasty. Aesthetic Plast Surg 2004;28:445-55.

2. Jung DH, Moon HJ, Choi SH, et al. Secondary rhinoplasty of the Asian nose: correction of the contracted nose. Aesthetic Plast Surg 2004;28:1-7.

3. Moon KC, Han SK. Surgical anatomy of the Asian nose. Facial Plast Surg Clin North Am 2018;26:259-68.

4. Kim IS. Augmentation rhinoplasty using silicone implants. Facial Plast Surg Clin North Am 2018;26:285-93.

5. Kim DA, Jeong JY, Oh SH. Bulbous tip correction focusing on skin soft tissue envelope in Asian rhinoplasty. Arch Aesthetic Plast Surg 2014;20:140-7.

6. Kerolus JL, Nassif PS. Treatment protocol for compromised nasal skin. Facial Plast Surg Clin North Am 2019;27:505-11.

7. Lee HM, Kim CK, Kim SH, et al. Fibroareolar tissue wrap-around flap for tip plasty in rhinoplasty in Asians. Arch Aesthetic Plast Surg 2016; 22:117-21.

8. Benvenuti D. Necrosis of the nasal tip. Plast Reconstr Surg 1995;96: 223-4.

9. Erdogan B, Tuncel A, Adanali G, et al. Augmentation rhinoplasty with dermal graft and review of the literature. Plast Reconstr Surg 2003;111: 2060-8.

10. Kim JH, Song JW, Park SW. Contracted nose correction with dermofat and conchal cartilage. Plast Reconstr Surg Glob Open 2017;5:e1272.

11. Kim YK. Augmentation rhinoplasty using dermofat graft. J Korean Soc Aesthetic Plast Surg 1999;5:33-40.

12. Na DS, Jung SW, Kook KS, et al. Augmentation rhinoplasty with dermofat graft \& fat injection. J Korean Soc Plast Reconstr Surg 2011;38: 53-62. 